\title{
Appearance of COVID-19 pneumonia on 1.5 T TrueFISP MRI
}

\author{
Aspectos da pneumonia por COVID-19 na ressonância magnética 1.5 T TrueFISP
}

\author{
Judith Eva Spiro ${ }^{1, a}$, Adrian Curta $^{1, b}$, Shiwa Mansournia ${ }^{1, c}$, Constantin Arndt Marschner ${ }^{1, d}$, Stefan Maurus ${ }^{1, e}$, \\ Ludwig Thomas Weckbach ${ }^{2, f}$, Dennis Martin Hedderich ${ }^{3, g}$, Julien Dinkel ${ }^{1,4,5, h}$
}

1. Department of Radiology, University Hospital, LMU Munich, Munich, Germany. 2. Department of Medicine I, University Hospital, LMU Munich, Munich, Germany. 3. Department of Neuroradiology, Technical University of Munich, School of Medicine, Munich, Germany. 4. Comprehensive Pneumology Center (CPC-M), Member of the German Center for Lung Research (DZL), Munich, Germany. 5. Department of Radiology, Asklepios Lung Center Munich-Gauting, Gauting, Germany.

Correspondence: Dr. Judith E. Spiro. Department of Radiology, University Hospital, LMU Munich. Marchioninistr. 15, D-81377 Munich, Germany. Email: judith.spiro@med.uni-muenchen.de.

a. https://orcid.org/0000-0003-2560-2713; b. https://orcid.org/0000-0003-1056-2149; c. https://orcid.org/0000-0002-4082-2370;

d. https://orcid.org/0000-0002-2042-7322; e. https://orcid.org/0000-0003-0413-851X; f. https://orcid.org/0000-0002-0981-2246;

g. https://orcid.org/0000-0001-8994-5593; h. https://orcid.org/0000-0003-1239-6084.

Received 1 February 2021. Accepted after revision 16 March 2021.

How to cite this article:

Spiro JE, Curta A, Mansournia S, Marschner CA, Maurus S, Weckbach LT, Hedderich DM, Dinkel J. Appearance of CoVID-19 pneumonia on 1.5 T TrueFISP MRI. Radiol Bras. Jul/Ago;54(4):211-218

Abstract Objective: To evaluate the performance of $1.5 \mathrm{~T}$ true fast imaging with steady state precession (TrueFISP) magnetic resonance imaging (MRI) sequences for the detection and characterization of pulmonary abnormalities caused by coronavirus disease 2019 (COVID-19). Materials and Methods: In this retrospective single-center study, computed tomography (CT) and MRI scans of 20 patients with COVID-19 pneumonia were evaluated with regard to the distribution, opacity, and appearance of pulmonary lesions, as well as bronchial changes, pleural effusion, and thoracic lymphadenopathy. McNemar's test was used in order to compare the COVID19-associated alterations seen on CT with those seen on MRI.

Results: Ground-glass opacities were better visualized on CT than on MRI ( $p=0.031)$. We found no statistically significant differences between CT and MRI regarding the visualization/characterization of the following: consolidations; interlobular/intralobular septal thickening; the distribution or appearance of pulmonary abnormalities; bronchial pathologies; pleural effusion; and thoracic lymphadenopathy.

Conclusion: Pulmonary abnormalities caused by COVID-19 pneumonia can be detected on TrueFISP MRI sequences and correspond to the patterns known from CT. Especially during the current pandemic, the portions of the lungs imaged on cardiac or abdominal MRI should be carefully evaluated to promote the identification and isolation of unexpected cases of COVID-19, thereby curbing further spread of the disease.

Keywords: Thorax; Tomography, spiral computed; Magnetic resonance imaging; Pneumonia; Coronavirus infections.

Resu mo Objetivo: Avaliar o desempenho da ressonância magnética (RM) de 1,5 T true fast imaging with steady state precession (TrueFISP) para detecção e caracterização de anormalidades pulmonares causadas por doença do coronavírus 2019 (COVID-19).

Materiais e Métodos: Neste estudo retrospectivo unicêntrico, imagens de tomografia computadorizada (TC) e RM de 20 pacientes com pneumonia COVID-19 foram avaliadas em relação a distribuição, opacidade e forma das lesões pulmonares, anormalidades brônquicas, derrame pleural e linfadenopatia torácica. 0 teste de McNemar foi usado para comparar os achados associados à COVID-19 na TC e na RM.

Resultados: As opacidades em vidro fosco foram mais bem visualizadas na TC do que na RM ( $p=0,031)$. Não foram encontradas diferenças estatisticamente significantes entre TC e RM em relação aos outros aspectos, ou seja, visualização de consolidações e espessamento septal interlobular/intralobular, distribuição ou forma de anormalidades pulmonares, doenças brônquicas, derrame pleural ou linfadenopatia torácica.

Conclusão: As anomalias pulmonares causadas pela pneumonia por COVID-19 podem ser detectadas nas sequências TrueFISP e correspondem aos padrões conhecidos da TC. Especialmente em face da pandemia atual, as porções de imagem dos pulmões na RM cardíaca ou abdominal devem ser cuidadosamente avaliadas para apoiar a identificação e o isolamento de casos inesperados de COVID-19 e, assim, conter a disseminação.

Unitermos: Tórax; Tomografia computadorizada, espiral; Ressonância magnética; Pneumonia; Infecções por coronavírus.

\section{INTRODUCTION}

Coronavirus disease 2019 (COVID-19) was first described in December 2019 in the city of Wuhan, China. Since then, infection with severe acute respiratory syndrome coronavirus 2 (SARS-CoV-2) has been spreading rapidly around the globe, causing a pandemic that, by the end of January 2021, resulted in more than 100 million confirmed cases and over 2 million confirmed deaths ${ }^{(1)}$.

Approximately $18 \%$ of people infected with SARS CoV2 are asymptomatic, the remainder developing symptoms 
such as fever, cough, and shortness of breath ${ }^{(2)}$. The last symptom in particular can be attributed to pneumonia caused by SARS-CoV-2, which in some patients has a severe course and may lead to death, especially in individuals with pre-existing medical conditions and in the elderly ${ }^{(2,3)}$. In addition, complications such as pulmonary artery embolism and myocardial injury worsen the prognosis of affected patients $^{(4,5)}$.

The current gold standard for diagnosing COVID-19 is pathogen detection using reverse transcriptase polymerase chain reaction (RT-PCR) of nose/throat swab or sputum samples. However, computed tomography (CT) also plays an important role because of its high sensitivity for the detection of atypical pneumonia ${ }^{(2,6)}$. A large number of studies have described the morphology of COVID-19 pneumonia on chest CT images. In the early stage of the infection, it typically includes bilateral, multilobar ground-glass opacities, predominantly in the subpleural space and lung bases. In the later stages, consolidations and linear opacities can be observed, as can thickened interlobular and intralobular septa, as well as the mosaic (crazy-paving) pattern and the reverse halo sign ${ }^{(7-9)}$.

Because COVID-19 is highly contagious and is transmitted through droplets, early identification and immediate isolation of infected individuals are of great importance in containing the pandemic. This procedure is complicated by the fact that many patients who are asymptomatic or have nonspecific symptoms are unaware of their infection and unwittingly spread the virus ${ }^{(2,10,11)}$. It is therefore particularly important to identify and isolate those patients whenever possible. Radiologists play a key role because signs of COVID-19 pneumonia may be incidental findings on radiological examinations ${ }^{(12,13)}$.

Although the potential of magnetic resonance imaging (MRI) for pulmonary imaging has been highlighted in a variety of studies, the suitability of MRI for the detection of COVID-19 pneumonia in particular has only occasionally been investigated ${ }^{(12-19)}$. Given that standard cardiac MRI protocols usually include sequences that cover both lungs, the examination is well suited for the detection of asymptomatic patients with pulmonary manifestations of COVID-19. We therefore evaluated whether pulmonary abnormalities caused by COVID-19 pneumonia can be seen on cardiac MRI and whether MRI scans show the pattern typically seen on CT.

\section{MATERIALS AND METHODS}

This was a retrospective single-center study. The study was approved by the research ethics committee of our institution. Due to the retrospective nature of the study, the requirement for written informed consent was waived.

\section{Study population}

We included patients with RT-PCR-confirmed COVID-19 who had undergone chest CT and cardiac MRI between March 18 and May 5, 2020. In all patients, the indication for performing cardiac MRI was an elevated troponin $\mathrm{T}$ level on a high-sensitivity troponin $\mathrm{T}$ assay, assuming that coronary artery disease had been excluded. Significant coronary artery disease was ruled out by coronary CT angiography or cardiac catheter examination within the last 24 months. A total of 20 patients (18 males and 2 females) met the inclusion criteria. The mean age was $64.8 \pm 13.9$ years (range, $27-82$ years). Two patients had a reduced left ventricular ejection fraction $(27 \%$ and $29 \%$, respectively), and another patient presented with sinus tachycardia $(117 \mathrm{bpm})$. The other patients did not show signs of cardiac dysfunction. Clinical and imaging findings were obtained from patient medical records. In cases in which more than one CT scan of the chest was acquired, the scan chronologically closest to the date of the MRI examination was analyzed.

\section{Chest CT}

Fifteen CT examinations with low-dose protocols were performed to evaluate pulmonary changes due to COVID19 pneumonia. Four contrast-enhanced CT scans were performed to rule out pulmonary embolism. One CT scan was performed for image-guided positioning of a chest tube due to large pleural effusion. Eighteen CT scans were performed on a Somatom Definition AS+ scanner (Siemens Healthineers, Erlangen, Germany), one was performed on a Somatom Force scanner (Siemens Healthineers) and one was performed on an Optima CT660 scanner (GE Healthcare, Milwaukee, WI, USA) scanner. The scan protocols were as follows: mean tube current of $93.4 \pm 66.1 \mathrm{mAs}$ (range, 37-232 mAs); mean tube voltage of $108.0 \pm 12.0$ $\mathrm{kVp}$ (range, 80-120 kVp); mean slice thickness of $1.7 \pm 0.9$ $\mathrm{mm}$ (range, 1-3 mm); mean volume CT dose index of 3.9 $\pm 1.3 \mathrm{mGy}$ (range, 0.8-15.3 $\mathrm{mGy}$ ); and mean dose-length product $(\mathrm{mGy} \times \mathrm{cm})$ of $131.6 \pm 102.0$ (range, 26-495).

\section{Thoracic MRI}

All MRI examinations were performed in the setting of suspected myocardial injury and in a Magnetom Aera 1.5 T scanner (Siemens Healthineers). The standard cardiac protocol at our department includes coronal, axial, and sagittal true fast imaging with steady state precession (TrueFISP) sequences covering the entire thorax, in order to evaluate the pulmonary changes. The scan parameters of the sequences were as follows: echo time, $1.38-1.65 \mathrm{~ms}$; repetition time, $441.59 \mathrm{~ms}$; flip angle, $80^{\circ}$; slice thickness, $6 \mathrm{~mm}$; field of view, $340 \times 276 \mathrm{~mm}$; and matrix, $256 \times 178$.

\section{Image analysis}

Two radiologists (with 7 and 14 years of experience, respectively) reviewed the chest CT and MRI scans on a picture archiving and communication system workstation (Syngo Studio version VB36E; Siemens Healthcare, Erlangen, Germany). Discrepancies were resolved by consensus. 
To avoid memory bias, the analyses of the CT and MRI scans were conducted with a two-week interval between them.

All scans were evaluated in axial, coronal, and sagittal orientation. For evaluation of CT images, lung window settings (width, $1600 \mathrm{HU}$; level, $-600 \mathrm{HU}$ ) and soft-tissue window settings (width, $300 \mathrm{HU}$; level, $40 \mathrm{HU}$ ) were applied.

Pulmonary findings on CT images were described according to the Fleischner Society glossary of terms for thoracic imaging ${ }^{(20)}$. Lung abnormalities observed on MRI were described according to the current literature on the subject, which adopts the CT terminology outlined in the Fleischner Society glossary. For instance, we defined ground-glass opacities as a hazy increase in signal intensity in the lung, with preserved bronchial and vascular $\operatorname{margins}^{(14,16-19,21,22)}$. The two readers evaluated the distribution of the pulmonary findings (unilateral vs. bilateral; unilobar vs. multilobar; affected lobes; involvement of upper, middle, and lower zones; peripheral or central lung involvement; anterior or posterior lung involvement; and subpleural sparing), as well as their opacity (groundglass opacities, consolidation, and interlobular/intralobular septal thickening) and appearance (patchy/segmental, rounded, crazy-paving, halo/reversed halo, linear/reticular, and air bronchogram). The readers also evaluated the bronchial changes (bronchiectasis, bronchial wall thickening, and mucus plugging), as well as looking for pleural effusion and thoracic lymphadenopathy. Regarding distribution, the inner two thirds and the outer third of the lung were defined as the central zone and the peripheral zone, respectively. A virtual horizontal line passing through the middle of the lung in the axial plane separated the anterior zone from the posterior zone, and two additional virtual horizontal lines dividing the lungs into equal thirds in the coronal plane delineated the upper, middle, and lower zones. The MRI findings were compared with the CT findings and with those in the current literature on COVID-19.

\section{Statistical analysis}

Statistical analysis was performed with the SPSS Statistics software package, version 25.0 (IBM Corp., Armonk, NY, USA). Quantitative variables are expressed as mean \pm standard deviation and range, and categorical variables are expressed as absolute and relative frequencies. McNemar's test was used in order to compare COVID19-associated findings on CT and MRI scans. The level of significance was set at $\alpha=0.05$.

\section{RESULTS}

Twenty patients with RT-PCR-proven COVID-19, all of whom had undergone CT and MRI examination of the chest between March 18 and May 5, 2020 at our institution, met the inclusion criteria. Demographic data and
Table 1-Demographic data and clinical symptoms of the patients included, at first admission.

\begin{tabular}{lc}
\hline Variable & $(\mathrm{N}=20)$ \\
\hline Gender, $\mathrm{n}(\%)$ & $18(90)$ \\
Male & $2(10)$ \\
Female & $13.9(27-82)$ \\
Age (years), mean \pm SD (range) & \\
Symptoms, $\mathrm{n}(\%)$ & $14(70)$ \\
Fever & $12(60)$ \\
Cough & $10(50)$ \\
Fatigue & $6(30)$ \\
Dyspnea & $4(20)$ \\
Nausea/emesis & $3(15)$ \\
Headache & $2(10)$ \\
Diarrhea & $1(5)$ \\
Abdominal pain & $1(5)$ \\
Chest pain & \\
\hline
\end{tabular}

clinical symptoms of the study population at first admission are presented in Table 1.

The mean interval between the first RT-PCR that was positive for SARS-CoV-2 and the CT examination was $8.1 \pm$ 11.7 days (range, -3 days [CT performed prior to RT-PCR] to 40 days). The mean interval between the first positive RT-PCR and the MRI examination was $17.2 \pm 11.7$ days (range, 4-39 days). The mean interval between CT and MRI was $9.0 \pm 9.0$ days (range, -7 days [MRI performed prior to CT] to 27 days). Twelve patients underwent only one MRI examination, and eight underwent an additional (follow-up) MRI, at an average of $42.0 \pm 7.4$ days (range, 27-51 days) after the first.

\section{Imaging findings}

All 20 patients showed pulmonary abnormalities on CT scans, as well as on MRI scans. The distribution and appearance of the pulmonary findings on CT and MRI are presented in Table 2 . The only significant difference between the two methods was that ground-glass opacities were visualized less often on MRI scans than on CT scans $(p=0.031)$. Bronchiectasis, bronchial wall thickening, and mucus plugging were seen on CT in 5\%, $15 \%$, and $15 \%$ of the patients, respectively, and on MRI in 5\%, $5 \%$, and $5 \%$, respectively. Thoracic lymphadenopathy and pleural effusions were seen on $30 \%$ and $35 \%$ of the CT scans, respectively, compared with $30 \%$ and $40 \%$ of the MRI scans, respectively. The septal thickening and crazypaving pattern seen on $20 \%$ of the CT scans was not detected on MRI scans, whereas linear/reticular pulmonary opacities were visualized almost as often on CT as on MRI (in $85 \%$ and $80 \%$ of the cases, respectively). Representative pulmonary findings and comparisons between CT and MRI findings are presented in Figures 1 and 2.

As previously mentioned, eight patients underwent follow-up MRI, which showed that the pulmonary opacities had decreased in size, and quantity. Only one of those 
Table 2-Distribution and appearance of thoracic findings on CT and MRI scans of patients with COVID-19 pneumonia $(\mathrm{N}=20)$.

\begin{tabular}{|c|c|c|c|}
\hline Parameter & $\begin{array}{c}\text { CT } \\
\text { n (\%) }\end{array}$ & $\begin{array}{c}\text { MRI } \\
\text { n (\%) }\end{array}$ & $P$-value \\
\hline \multicolumn{4}{|l|}{ Location } \\
\hline Unilateral & $1(5)$ & $2(10)$ & 1.000 \\
\hline Bilateral & 19 (95) & $18(90)$ & 1.000 \\
\hline Unilobar & $1(5)$ & 2 (10) & 1.000 \\
\hline Multilobar & $19(95)$ & $18(90)$ & 1.000 \\
\hline Upper zone & $16(80)$ & $17(85)$ & 1.000 \\
\hline Middle zone & $19(95)$ & $19(95)$ & 1.000 \\
\hline Lower zone & $20(100)$ & 19 (95) & 1.000 \\
\hline Anterior zone & $18(90)$ & $17(85)$ & 1.000 \\
\hline Posterior zone & $20(100)$ & $20(100)$ & 1.000 \\
\hline Central zone & $18(90)$ & $14(70)$ & 0.219 \\
\hline Peripheral zone & $20(100)$ & $20(100)$ & 1.000 \\
\hline Subpleural sparing & $10(50)$ & $5(25)$ & 0.180 \\
\hline \multicolumn{4}{|l|}{ Affected lobes } \\
\hline Right upper & $18(90)$ & $15(75)$ & 0.250 \\
\hline Middle & $16(80)$ & $13(65)$ & 0.250 \\
\hline Right lower & 19 (95) & $20(100)$ & 1.000 \\
\hline Left upper & $16(80)$ & $16(80)$ & 1.000 \\
\hline Left lower & $19(95)$ & $18(90)$ & 1.000 \\
\hline \multicolumn{4}{|l|}{ Opacity } \\
\hline Ground-glass & $20(100)$ & $14(70)$ & 0.031 \\
\hline Consolidation & $19(95)$ & 20 (100) & 1.000 \\
\hline Inter-/intralobular septal thickening & $4(20)$ & $0(0)$ & 0.125 \\
\hline \multicolumn{4}{|l|}{ Appearance } \\
\hline Patchy/segmental & $20(100)$ & $20(100)$ & 1.000 \\
\hline Reversed halo sign & $3(15)$ & $4(20)$ & 1.000 \\
\hline Rounded & $4(20)$ & $1(5)$ & 0.250 \\
\hline Linear/reticular opacities & $17(85)$ & $16(80)$ & 1.000 \\
\hline Crazy-paving pattern & $4(20)$ & $0(0)$ & 0.125 \\
\hline Air bronchogram & $7(35)$ & $4(20)$ & 0.250 \\
\hline \multicolumn{4}{|l|}{ Bronchial changes } \\
\hline Bronchiectasis & $1(5)$ & $1(5)$ & 1.000 \\
\hline Bronchial wall thickening & $3(15)$ & $1(5)$ & 0.500 \\
\hline Mucus plugging & $3(15)$ & $1(5)$ & 0.500 \\
\hline \multicolumn{4}{|l|}{ Other } \\
\hline Pleural effusion & $7(35)$ & $8(40)$ & 1.000 \\
\hline Hilar/mediastinal lymphadenopathy & $6(30)$ & $6(30)$ & 1.000 \\
\hline
\end{tabular}

patients underwent follow-up CT, and the follow-up data were therefore not included in the statistical analysis. However, representative images are shown in Figure 3.

\section{DISCUSSION}

The results of the present study indicate that it is possible to use TrueFISP MRI sequences to detect pneumonia caused by SARS-CoV-2 infection. On CT and MRI, the predominant findings were patchy/segmental and linear/reticular ground-glass opacities and consolidations, with a multilobar, bilateral distribution, in the peripheral zone of the lungs. The right lower lobe was most often affected. Regarding the distribution and appearance of the pulmonary findings, as well as bronchial changes, pleural effusion, and thoracic lymphadenopathy, no statistically

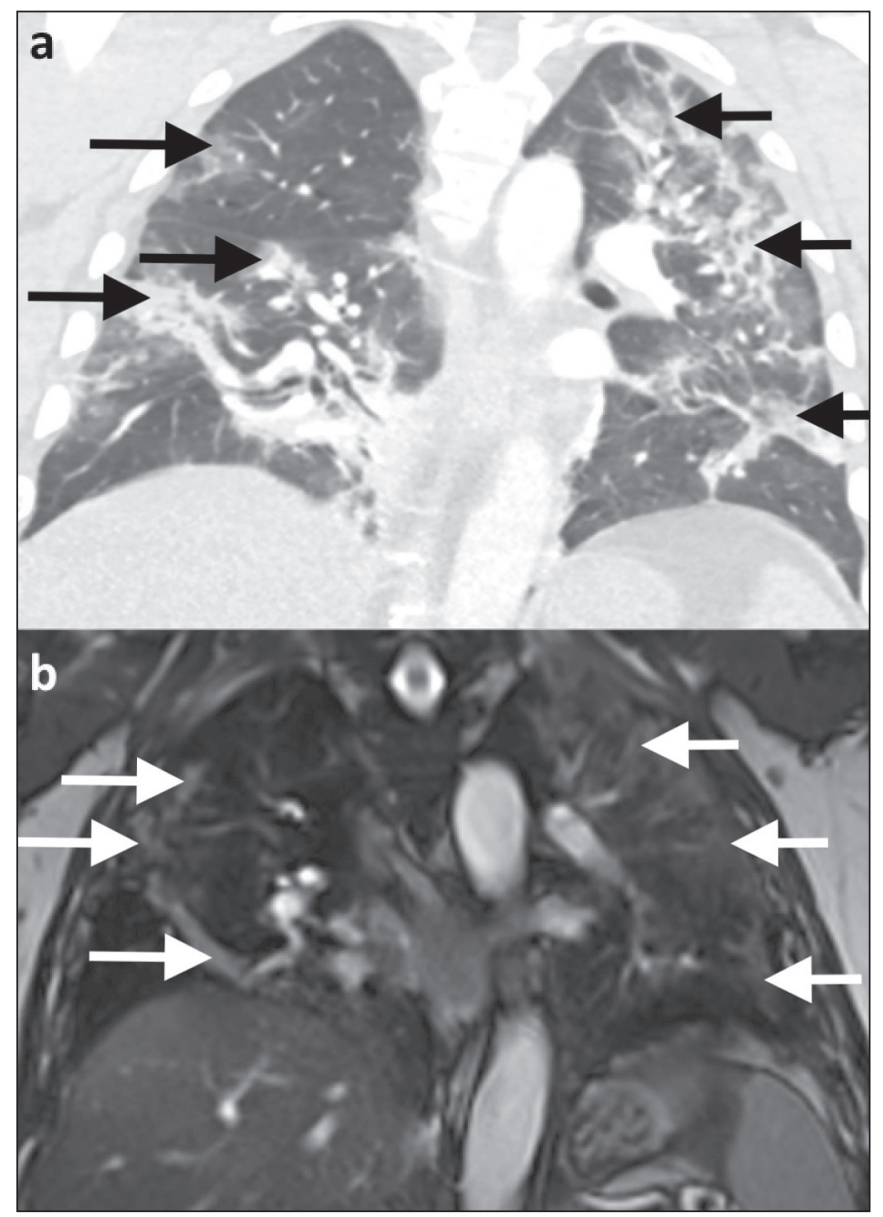

Figure 1. Ability of 1.5 T TrueFISP MRI to show the distribution of pulmonary findings. CT and MRI scans (a and $\mathbf{b}$, respectively) showing patchy and linear consolidations and ground-glass opacities in the upper, middle, and lower lobes, as well as in the central and peripheral zones of the lungs (arrows). The CT scan was acquired on the day of COVID-19 confirmation by RT-PCR. MRI was performed 17 days after the CT.

significant difference was found between the MRI and CT scans. Although ground-glass opacities were visualized significantly less often on MRI scans than on CT scans, there were no statistically significant differences in terms of the visualization of consolidations and interlobular/intralobular septal thickening.

Our results are in line with those of other studies. The appearance and distribution of pulmonary abnormalities in our sample (i.e., the predominance of multilobar, peripheral ground-glass opacities and consolidations with a patchy and linear appearance) are typical manifestations of COVID-19 pneumonia described in the current literature ${ }^{(7-9,23)}$. The frequency of bronchial pathologies in our patient sample also coincides with that reported in other recent studies. Bernheim et al. ${ }^{(7)}$ reported bronchiectasis, bronchial wall thickening, and mucus plugging in $0.8 \%$, $11.6 \%$, and $0.8 \%$ of the patients evaluated, respectively, and Inui et al. ${ }^{(23)}$ found bronchial abnormalities in a total of $28 \%$. In the present study, thoracic lymphadenopathy and pleural effusions were more common than has been reported in other studies on the subject ${ }^{(7,9,23)}$. However, 


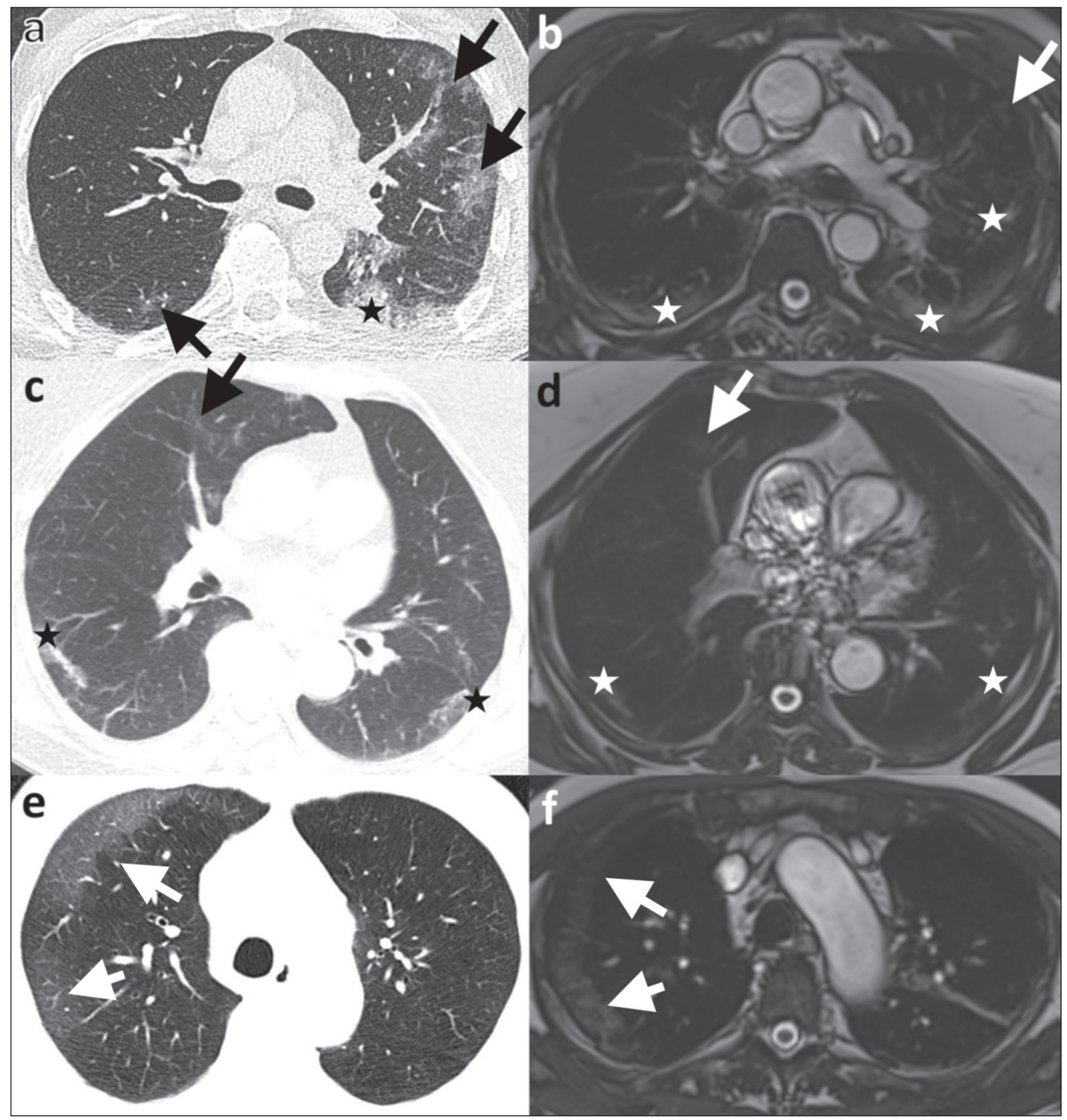

Figure 2. Ability of 1.5 T TrueFISP MRI to detect consolidations and ground-glass opacities. CT scan (a) showing peripheral ground-glass opacities of the left upper and right lower lobe (arrows), as well as consolidations of the left lower lobe (star), in a patient who was diagnosed with COVID-19 via RT-PCR two days later. MRI of the chest (b), performed 6 days after CT, showing consolidations in the lower lobes and left upper lobe (stars), with barely visible ground-glass opacities (arrow). Chest CT (c) of another patient, performed 14 days after confirmation of COVID-19 by RT-PCR, showing ground-glass opacities (arrow) and consolidations (stars), which are almost equally as visible on an MRI scan (d) acquired 2 days earlier. In another patient with CoVID-19, ground-glass opacities with subpleural sparing in the right upper lobe (arrows) are present on a CT scan (e) performed on the day of COVID-19 confirmation by RT- PCR, as well as on an MRI scan (f) acquired 4 days later.

the current knowledge on COVID-19 pneumonia suggests that such findings are indicative of greater severity ${ }^{(2)}$. All of the patients in our sample had suspected myocardial injury and therefore a rather severe disease course, which could explain the higher prevalence of thoracic lymphadenopathy and pleural effusion in our sample.
Our comparison of CT and MRI revealed broad agreement between the two modalities, except for the visualization of ground-glass opacities: We detected pulmonary abnormalities on all scans and found no statistically significant difference between CT and MRI concerning the distribution of pulmonary findings in patients with COVID-19 


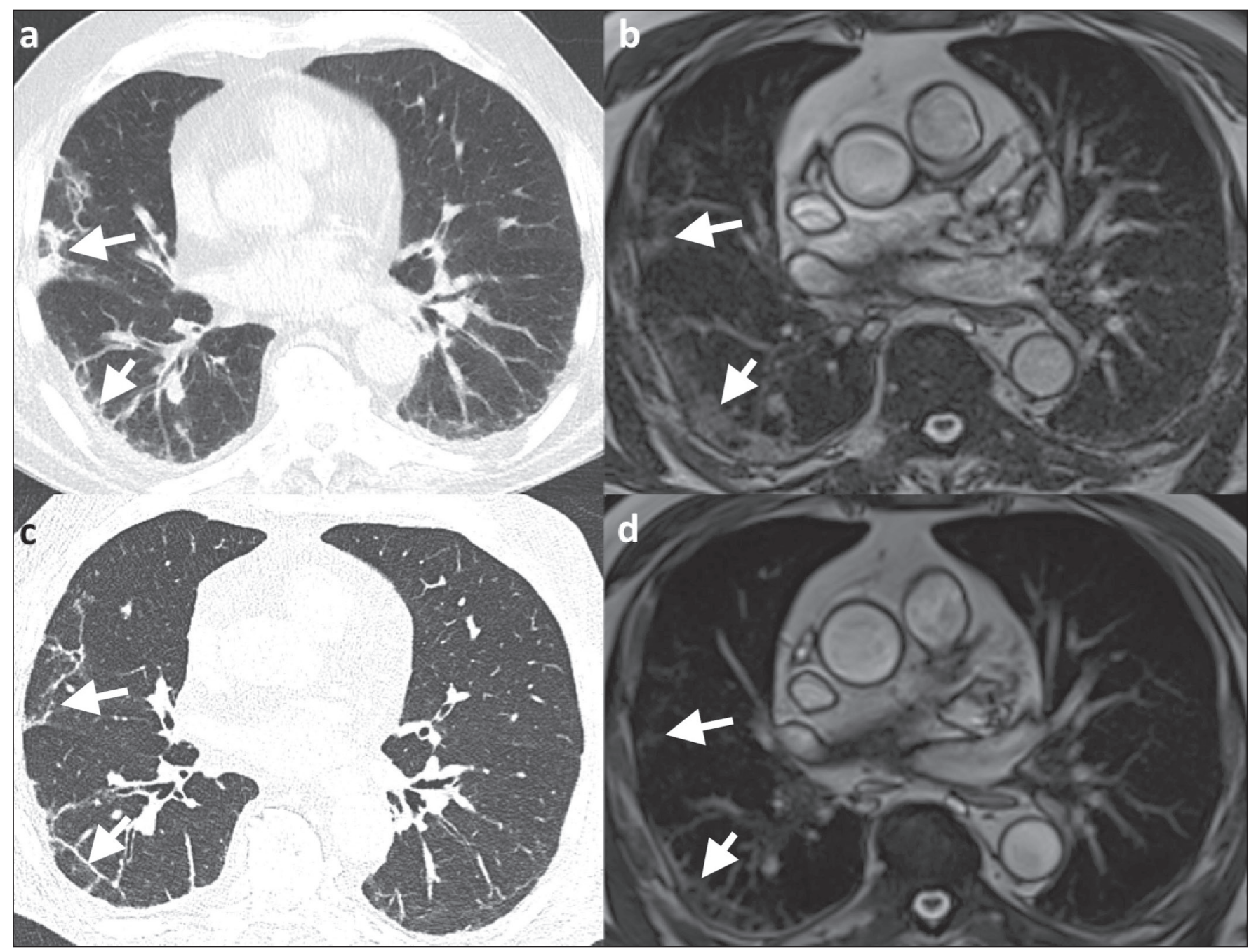

Figure 3. Appearance of pulmonary changes in COVID-19 pneumonia on 1.5 T TrueFISP MRI over time. Chest CT (a) of a patient who was diagnosed with COVID-19 via RT-PCR 28 days earlier, showing patchy and reticular consolidations in the middle lobe and right lower lobe (arrows). The opacities are almost equally as visible on an MRI scan (b) performed 8 days later. Follow-up CT scan (c) and follow-up MRI scan (d) acquired 56 and 59 days, respectively, after the RT-PCR almost equally show the reduction of the opacities to linear residuals (arrows).

pneumonia. Accordingly, Attenberger et al., evaluating the suitability of 3-T MRI for the diagnosis of pneumonia in patients with neutropenia, reported that MRI and highresolution CT are comparable in terms of overall lesion localization. Although MRI produced some false-positive and false-negative results in that study, those results did not alter the treatment strategy, because the pulmonary abnormalities were disseminated in all patients ${ }^{(16)}$.

In our sample, CT was slightly better at revealing septal thickening and the crazy-paving pattern than was MRI, although the differences were not statistically significant, whereas the two methods were comparable in terms of their ability to detect linear/reticular opacities. Linear/ reticular opacities are usually more pronounced than are septal thickening and the crazy-paving pattern, and previous studies have shown that, when compared with CT, thoracic MRI has lower sensitivity for the detection of small pulmonary lesions ${ }^{(5,9)}$. In addition, linear/reticular opacities represent a stage of COVID-19 pneumonia later than that represented by septal thickening and the crazy- paving pattern ${ }^{(11)}$. Therefore, the discrepancy in the identification of linear/reticular opacities, septal thickening, and the crazy-paving pattern on MRI scans is presumably attributable to the lower sensitivity of TrueFISP MRI sequences for the identification of subtle pulmonary abnormalities and the time interval between the acquisition of CT and MRI scans in our study.

Regarding opacities, the authors of several other studies have also reported that the sensitivity of MRI is equivalent to that of CT for the detection of consolidations, although not for ground-glass opacities, some of which cannot be seen on MRI ${ }^{(16,24)}$. Recently, Singh et al. ${ }^{(21)}$ evaluated the diagnostic accuracy of MRI for respiratory infections in immunocompromised patients. Their MRI protocol, like ours, included a TrueFISP sequence, and the authors found it to have a sensitivity of $100 \%$ for the detection of consolidations and of only $16.6 \%$ for the detection of ground-glass opacities. Possible explanations for this phenomenon are the relatively low proton density of ground-glass opacities relative to that of consolidations, 
signal loss due to $2_{2}^{*}$ decay, and cardiac motion. Promising results regarding the visualization of ground-glass opacities have been reported for low-field MRI, the low susceptibility of which results in higher signal intensity of lung parenchyma, as well as for respiratory-gated ultra-short echo time (UTE) MRI, which is less susceptible to T2* decay and motion artifacts than are other sequences ${ }^{(22,25)}$. Yang et al. ${ }^{(14)}$ examined the potential of UTE-MRI and CT for assessing COVID-19 pneumonia and reported that the two modalities provide similar image quality and high concordance for assessing the representative image findings. In particular, the lesion-based agreement between CT and UTE-MRI for evaluating ground-glass opacities was excellent (kappa: 0.815). For interlobular/intralobular septal thickening and the crazy-paving pattern, which were not visible on the MRI scans in our study, the level of intermethod agreement was moderate (kappa: 0.564) in the Yang et al. ${ }^{(14)}$ study. Heiss et al. ${ }^{(18)}$ reported a case in which persistent pulmonary abnormalities after COVID-19, including ground-glass opacities, were precisely visualized on low-field (0.55-T) MRI. The results reported by Heiss et al. ${ }^{(18)}$ and Yang et al. ${ }^{(14)}$ are promising with regard to using MRI for the diagnosis and surveillance of COVID-19 pneumonia in the future. However, the use of complex, nonstandard sequences and scanner systems for the optimal detection and characterization of subtle pulmonary abnormalities like ground-glass opacities was not the aim of our study. Rather, we wanted to determine whether COVID-19 pneumonia can be detected on standard MRI protocols, without the acquisition of dedicated lung sequences, carried out for reasons other than the investigation of pneumonia.

Although we were able to detect bronchial wall thickening and mucus plugging more often on CT than on MRI (in $15 \%$ and $5 \%$ of the cases, respectively), the difference did not reach statistical significance. In our sample, bronchiectasis was seen on one CT scan and one MRI scan. Arslan et al. ${ }^{(26)}$ reported comparable results for MRI in patients with primary immunodeficiency. The similar performance of CT and MRI for the detection of pleural effusion and thoracic lymphadenopathy, as observed in our study, has also been described in previous studies ${ }^{(15,22,27)}$.

Our study has some limitations. Among the patients included, the course of the disease was rather severe, because they all had suspected myocardial injury. That probably led to the extensive pulmonary abnormalities, which are not representative of all affected individuals. In addition, the relatively small number of patients limits the statistical power of the analyses. Nevertheless, our findings are in line with those of previous studies, which is why we assume that our results are reliable. Another limitation are the relatively long and varied time intervals between RTPCR tests and imaging, as well as between CT and MRI examinations. Because the presentation, location, and extent of pulmonary abnormalities in COVID-19 pneumonia change, the comparability between CT and MRI findings is reduced over time ${ }^{(7)}$. For a lesion-based approach (i.e., a direct comparison of the appearance of single lesions, especially ground-glass opacities), a shorter time interval between the CT and MRI examinations would be essential. In this context, state-of-the-art sequences dedicated to lung imaging should be examined. The aim of our study, however, was to evaluate whether the pulmonary manifestations of COVID-19 pneumonia are generally visible and basically show the typical pattern described for CT on the widely used and available TrueFISP MRI sequences. As in larger studies, the CT and MRI scans in our study showed the typical appearance of COVID-19 pneumonia on CT, in all stages of the disease ${ }^{(9)}$, and the MRI findings in our study not only correlated well with those of other studies but also with the CT findings. We were therefore able to show that in the setting of the pandemic, pulmonary abnormalities due to infection with SARS-CoV-2 can be incidentally recognized as a COVID-19 pattern on MRI scans performed for other reasons.

\section{CONCLUSION}

In conclusion, we were able to show that the pulmonary changes caused by COVID-19 pneumonia on TrueFISP MRI sequences are consistent with the patterns known from CT, and that such abnormalities can be detected on cardiac MRI. Given the special setting of the current pandemic, MRI examinations that show portions of the lungs should be carefully evaluated by the attending radiologists to promote the identification and isolation of unknown COVID-19 cases, thus curbing further spread of the disease. The results obtained encourage further detailed research to determine the value of lung MRI for the diagnosis of COVID-19 pneumonia.

\section{REFERENCES}

1. World Health Organization. Coronavirus disease (COVID-19) pandemic. [Internet]. 2020 [cited 2021 Jan 29]. Available from: https:// www.who.int/emergencies/diseases/novel-coronavirus-2019.

2. Siordia JA Jr. Epidemiology and clinical features of COVID-19: a review of current literature. J Clin Virol. 2020;127:104357.

3. Zheng Z, Peng F, Xu B, et al. Risk factors of critical \& mortal COVID-19 cases: a systematic literature review and meta-analysis. J Infect. 2020;81:e16-25.

4. Tomasoni D, Italia L, Adamo M, et al. COVID-19 and heart failure: from infection to inflammation and angiotensin II stimulation. Searching for evidence from a new disease. Eur J Heart Fail. 2020;22:957-66.

5. Poyiadji N, Cormier P, Patel PY, et al. Acute pulmonary embolism and COVID-19. Radiology. 2020;297:E335-8.

6. Waller JV, Kaur P, Tucker A, et al. Diagnostic tools for coronavirus disease (COVID-19): comparing CT and RT-PCR viral nucleic acid testing. AJR Am J Roentgenol. 2020;21 5:834-8.

7. Bernheim A, Mei X, Huang M, et al. Chest CT findings in coronavirus disease-19 (COVID-19): relationship to duration of infection. Radiology. 2020;295:200463.

8. Pan Y, Guan H, Zhou S, et al. Initial CT findings and temporal changes in patients with the novel coronavirus pneumonia $(2019-\mathrm{nCoV})$ : a study of 63 patients in Wuhan, China. Eur Radiol. 2020;30:3306-9. 
9. Salehi S, Abedi A, Balakrishnan S, et al. Coronavirus disease 2019 (COVID-19): a systematic review of imaging findings in 919 patients. AJR Am J Roentgenol. 2020;215:87-93.

10. Rothan HA, Byrareddy SN. The epidemiology and pathogenesis of coronavirus disease (COVID-19) outbreak. JAutoimmun. 2020; 109: 102433.

11. Rothe C, Schunk M, Sothmann P, et al. Transmission of 2019$\mathrm{nCoV}$ infection from an asymptomatic contact in Germany. N Engl J Med. 2020;382:970-1.

12. Fonseca EKUN, Chate RC, Sasdelli Neto R, et al. Pulmonary findings of COVID-19 identified at cardiac MRI. Radiol Cardiothorac Imaging. 2020;2:e200193.

13. Langenbach MC, Grosse-Hokamp N, Persigehl T, et al. MRI appearance of COVID-19 infection. Diagn Interv Radiol. 2020;26:377-8.

14. Yang S, Zhang Y, Shen J, et al. Clinical potential of UTE-MRI for assessing COVID-19: patient- and lesion-based comparative analysis. J Magn Reson Imaging. 2020;52:397-406.

15. Ekinci A, Uçarkus TY, Okur A, et al. MRI of pneumonia in immunocompromised patients: comparison with CT. Diagn Interv Radiol. $2017 ; 23: 22-8$.

16. Attenberger UI, Morelli JN, Henzler T, et al. 3 Tesla proton MRI for the diagnosis of pneumonia/lung infiltrates in neutropenic patients with acute myeloid leukemia: initial results in comparison to HRCT. Eur J Radiol. 2014;83:e61-6.

17. Ates OF, Taydas O, Dheir H. Thorax magnetic resonance imaging findings in patients with coronavirus disease (COVID-19). Acad Radiol. 2020;27:1373-8.

18. Heiss R, Grodzki DM, Horger W, et al. High-performance low field MRI enables visualization of persistent pulmonary damage after COVID-19. Magn Reson Imaging. 2021;76:49-51.

19. Torkian P, Rajebi H, Zamani T, et al. Magnetic resonance imaging features of coronavirus disease 2019 (COVID-19) pneumonia: the first preliminary case series. Clin Imaging. 2021;69:261-5.

20. Hansell DM, Bankier AA, MacMahon H, et al. Fleischner Society: glossary of terms for thoracic imaging. Radiology. 2008;246:697722.

21. Singh R, Garg M, Sodhi KS, et al. Diagnostic accuracy of magnetic resonance imaging in the evaluation of pulmonary infections in immunocompromised patients. Pol J Radiol. 2020;85:e53-61.

22. Ohno Y, Koyama H, Yoshikawa T, et al. Pulmonary high-resolution ultrashort TE MR imaging: comparison with thin-section standardand low-dose computed tomography for the assessment of pulmonary parenchyma diseases. J Magn Reson Imaging. 2016;43:512-32.

23. Inui S, Fujikawa A, Jitsu M, et al. Chest CT findings in cases from the cruise ship Diamond Princess with coronavirus disease 2019 (COVID-19). Radiol Cardiothorac Imaging. 2020;2:e2001 10.

24. Ozcan HN, Gormez A, Ozsurekci Y, et al. Magnetic resonance imaging of pulmonary infection in immunocompromised children: comparison with multidetector computed tomography. Pediatr Radiol. 2017;47:146-53.

25. Campbell-Washburn AE. 2019 American Thoracic Society BEAR cage winning proposal: lung imaging using high-performance lowfield magnetic resonance imaging. Am J Respir Crit Care Med. 2020; 201:1333-6.

26. Arslan S, Poyraz N, Ucar R, et al. Magnetic resonance imaging may be a valuable radiation-free technique for lung pathologies in patients with primary immunodeficiency. J Clin Immunol. 2016;36: 66-72.

27. Schaffer JM, Singh SK, Reitz BA, et al. Single- vs double-lung transplantation in patients with chronic obstructive pulmonary disease and idiopathic pulmonary fibrosis since the implementation of lung allocation based on medical need. JAMA. 2015;313:936-48.

\section{(c) BY}

